\title{
MAINTAINING IMMOBILISATION DEVICES ON TRAUMA PATIENTS DURING CHEST AND PELVIC X- RAY: A FEASIBILITY STUDY
}

Pål Johan Stokkeland ( $\square$ paasto@sshf.no)

Sørlandet Hospital Kristiansand

Research article

Keywords: Trauma, spinal immobilization, vacuum mattress, anthropomorphic phantom, conventional radiography, artefacts.

Posted Date: July 9th, 2019

DOl: https://doi.org/10.21203/rs.2.11154/v1

License: (c) (i) This work is licensed under a Creative Commons Attribution 4.0 International License.

Read Full License 


\section{Abstract}

BACKGROUND The aim of our study was to investigate the effect of different vacuum mattresses on standard resuscitation bay conventional radiography of chest and pelvis, especially regarding artefacts. METHODS We used a mobile X-ray machine to perform chest and pelvic conventional radiography on an anthropomorphic whole body phantom with a trauma transfer board, three different vacuum mattresses and without any stabilization device. The vacuum mattresses were investigated in activated, deactivated and stretched after deactivated states. Two radiologists assessed the artefacts independently. Agreement was measured using kappa coefficient. RESULTS All radiographs were of good technical quality and fully diagnostic. With the exception of one disagreed occurrence, artefacts were seen to hamper clinical judgement exclusively with activated vacuum mattresses. There was substantial agreement between the two radiologists on artefact assessment. The observed agreement was 0,82 with a kappa coefficient of 0,71 . The first vacuum mattress caused no artefacts hampering with clinical judgment. CONCLUSION Our study concludes that it is feasible to maintain some vacuum mattresses through resuscitation bay conventional radiography of chest and pelvis. They do not result in artefacts hampering with clinical judgment. Our vacuum mattress $\mathrm{nr} 1$ is recommendable for this purpose. Together with our previous findings our present results indicate that some vacuum mattresses may be used throughout the initial resuscitation bay assessment and CT examination.

\section{Background}

Most trauma systems and traumatic spinal injury guidelines mandate spinal stabilization from the site of injury to a radiological confirmation or refutal of spinal injury [1, 2]. Vacuum mattresses have been advocated for patients in need of prehospital spinal stabilization [3, 4]. A vacuum mattress (VM) is a fullbody sized device that is wrapped around the patient (Fig. 1). It contains small plastic beads that render the VM rigid upon activation (evacuation of air). A VM is thought to provide better stabilization and comfort, compared to the backboard $[4,5]$.

Norwegian national guidelines recommend the use of a VM for patients in need of prehospital spinal stabilization [6]. Therefore, Norwegian Emergency Department trauma teams will probably receive more patients where a VM is applied and activated.

Our previous study indicated that it is feasible to maintain spinal stabilization with certain VMs throughout a trauma CT examination [7]. Rather than removing the VM upon arrival at the hospital it may be desirable to maintain the VM for spinal stabilization throughout the resuscitation bay initial assessment. However, this necessitates that it is feasible to perform the initial assessment including chest and pelvic X-ray examinations with a VM in place. In order to perform a complete clinical assessment the VM must be temporarily deactivated.

We have not been able to identify prior studies of artefacts from modern VMs on resuscitation bay radiographs. The aim of our study was to investigate the effect of different VMs on standard 
resuscitation bay conventional radiography (CR) of chest and pelvis, especially regarding artefacts.

\section{Methods}

To cover all technical and practical issues, the study group included members covering the treatment chain from the trauma site (paramedic), emergency department (anaesthesiologist and Head of Trauma; ER nurse), trauma CR-acquisition (radiographer), interpretation (radiologists), and quality control (physicist).

\section{PHANTOM, IMMOBILISATION DEVICES AND TRAUMA TABLE}

We used an anthropomorphic whole body phantom (PBU-60, Kyoto Kagaku Co. LTD, Kyoto, Japan). The phantom resembles a small Caucasian adult patient with a weight of $50 \mathrm{~kg}$ and a height of $165 \mathrm{~cm}$ (Fig. 2). It contains a synthetic skeleton, soft tissue, liver, kidneys, and vessels (Fig. 2). We performed chest and pelvic radiography using three different vacuum mattresses (Table 1). We made sets of CR with the vacuum mattresses both moulded around the phantom and activated with the straps attached, then deactivated and lastly de-activated plus stretching the mattress in order to minimize folds. We repeated each set three times. Being the present protocol in our institution, we also did CR with a trauma transfer board. A reference image without any of the devices applied was acquired and made available during the evaluation for comparison. We used the trauma table (JI-2100, Jostedal Industrier, Jostedal, Norway) that is standard in our institution.

Table 1. List of immobilisation devices investigated

\section{CR PROTOCOL}

We performed the CR on a mobile X-ray machine (MobileDaRt Evolution, Shimadzu, Kyoto, Japan) with a flat panel detector (CXDI-55-C High Sensitivity Detector, Canon, Tokyo, Japan) with fixed tube potential and tube current (Chest $125 \mathrm{kV} / 4 \mathrm{mAs}$, Pelvis 78kV/25mAs).

\section{ASSESSMENTS}

Two specialists in radiology independently reviewed the trauma radiographs regarding artefacts and were blinded to the specific type of device. The order of the CRs was randomized by a pseudo random number generator (Mersenne-Twister shuffle generator from the NumPy computer package [8]) assigning each examination with a random patient name and number.

The artefacts were categorised as no artefacts (category 1), artefacts not impeding clinical judgement (category 2), artefacts impeding clinical judgement (category 3), and artefacts rendering the investigation 
unsuitable for clinical judgement (category 4) (examples of artefacts in Fig. 3). Artefacts not visualised inside the phantom were classified as category 2 . Artefacts lateral of the glenoid cavity, not affecting the lungs, where categorized as 2 in the chest radiographs.

Agreement between the two radiologists was assessed using Cohen's kappa test statistic. The degree of observer agreement indicated by kappa values was interpreted as: [0-0.2] slight agreement; (0.2-0.4] fair agreement; (0.4-0.6] moderate agreement; (0.6-0.8] substantial agreement; and (0.8-1.0] almost perfect agreement [9].

\section{Results}

All radiographs were of good technical quality and fully diagnostic.

The two radiologists agreed on 46 of 56 evaluations regarding artefacts (Table 2). The inter-observer agreement was 0,82 with a kappa coefficient of 0,71 (95\% confidence interval $0.55,0.87$ ). Apart from one single disagreed evaluation in VM 3, category 3 artefacts were seen exclusively with activated VMs. Category 3-and 4-artefacts were not seen with VM1 altogether.

Table 2

\section{Discussion}

We found substantial agreement between the two radiologists regarding the presence and category of artefacts. Overall the artefacts were subtle, especially with the VM deactivated.

We performed CR with activated VMs although this is not presently applicable to our knowledge - CR will probably be executed during the initial assessment, which includes clinical examination necessitating exposure of the patient. Therefore, the VM must be deactivated during this phase.

Category 3 artefacts were with one exception seen exclusively in activated VMs. The single exception applies to VM3; however it was interpreted as category 2 after stretching the VM. This indicates that all three VMs could be used with resuscitation bay $\mathrm{x}$-ray using present protocol at our institution. If the VM must be used activated, VM1 produced only category 2 artefacts when activated.

No added value was seen from stretching of the VM in the remaining results.

The CR with VMs was done without trauma transfer board, in accordance with the Norwegian guidelines. Our evaluation showed no artefacts from the trauma transfer board and artefacts with VMs de-activated are minor. Thus we have no indication that a combined use should result in major artefacts. This is, however, an assumption that should be further investigated.

We hypothesized that artefacts might be caused by mattress polymer surface folds, plastic valves and securing straps with plastic buckles as well as uneven distribution of the polystyrene beads. We chose to 
repeat each set of $\mathrm{CR}$ three times with mattress re-orientation, deflation and inflation between the sets to allow for potential redistribution of artefacts.

There are several limitations to our study. First, all radiographs were performed solely with the same mobile x-ray machine and trauma table. We cannot be certain that alternative setups would give similar results. Second, the phantom represents a small adult patient. As the mattress is uni-size we assume this will result in relatively more accentuated artefacts compared to radiographing with a larger patient. However, the opposite would be the case for children. Third, it was not possible to blind the interpreters completely to the generic type of device. However, they were blinded to manufacture type. Our radiologists are not familiar to immobilisation devices in daily practise and we have assumed they therefore do not carry any bias on device type. Fourth, our radiologists were not accustomed to interpreting radiographs of a phantom for artefacts so this may have affected the results.

Together with our previous findings [7] our present results indicate that some VMs may be used throughout the initial resuscitation bay assessment and CT examination. However, if instituting such a protocol the quality assurance should be rigid. In addition, such a protocol should perhaps be subject to a research project before general application.

\section{CONCLUSION}

Our results indicate that it is feasible to maintain some vacuum mattresses on trauma patients during standard resuscitation bay conventional radiography of the chest and pelvis. Two of the mattresses did not result in artefacts that impeded clinical judgement when de-activated.

\section{Abbrevations}

VM: Vacuum mattress

CT: Computed tomography

CR: Conventional radiography

ER: Emergency room

\section{Declarations}

\section{ETHICS APPROVAL AND CONSENT TO PARTICIPATE}

Not applicable.

\section{CONSENT FOR PUBLICATION}

Not applicable. 


\section{AVAILABILITY OF DATA AND MATERIALS}

DICOM images and acquired datasets will be made available from the corresponding author on reasonable request.

\section{CONFLICTS OF INTEREST}

SA has served as an advisor for AB Germa, Sweden and Kohlbrat \& Bunz GmbH, Austria but has gained no economic compensation or any other benefits. All other authors declare no competing interests, economic or otherwise.

\section{FUNDING}

This project was funded by Sørlandet Hospital.

\section{AUTHOR CONTRIBUTIONS}

PKH conceived the study. PJS, EA, MMB, AIM, CPGA, SA, and PKH designed the study. Data acquisition was performed by PJS, EA, AIM and CPGA. PJS and EA analysed and interpreted the data. PJS drafted the manuscript. All authors contributed to revision of the manuscript and approved the final version.

\section{ACKNOWLEDGEMENTS}

We wish to thank the University of Troms $\varnothing$ for lending us the phantom, radiologist Jon Bache Marthinsen, SSHF, for artefact assessments, and dr. Lars Webb for language assistance.

\section{References}

1. PHTLS Committee of NAEMT: Spinal Trauma. In PHTLS - Basic and Advanced Prehospital Trauma Life Support. 7th edition. Edited by McSwain NE. St. Louis, IL, USA: Mosby JEMS, Elsevier; 2011: 245289

2. American College of Surgeons Committee on Trauma: Spine and Spinal Cord Trauma. In Advanced Trauma Life Support for Doctors - Student Course Manual. 9th edition. Edited by Rotondo MF. Chicago, IL, USA: American College of Surgeons; 2012: 174-20

3. Luscombe MD, Williams JL: Comparison of a long spinal board and vacuum mattress for spinal immobilisation. Emerg Med J 2003;20:476-478.

4. Prasarn ML, Hyldmo PK, Zdziarski LA, Loewy E, Dubose D, Horodyski M, Rechtine GR: Comparison of the Vacuum Mattress versus the Spine Board Alone for Immobilization of the Cervical Spine Injured 
Patient: A Biomechanical Cadaveric Study. Spine (Phila Pa 1976) 2017;42:E1398-E1402.

5. Hamilton RS, Pons PT: The efficacy and comfort of full-body vacuum splints for cervical-spine immobilization. J Emerg Med 1996;14:553-559.

6. Kornhall DK, Jørgensen JJ, Brommeland T, et al. The Norwegian guidelines for the prehospital management of adult trauma patients with potential spinal injury. Scandinavian Journal of Trauma, Resuscitation and Emergency Medicine. 2017;25:2. doi:10.1186/s13049-016-0345-x.

7. Stokkeland PJ, Andersen E, Bjørndal MM, Mikalsen AM, Aslaksen S, Hyldmo PK. Maintaining immobilisation devices on trauma patients during CT: a feasibility study. Scandinavian Journal of Trauma, Resuscitation and Emergency Medicine. 2017;25:84. doi:10.1186/s13049-017-0428-3.

8. Jones E, Oliphant E, Peterson P, et al. SciPy: Open Source Scientific Tools for Python, 2001-, http://www.scipy.org/ Accessed 201a8-09-03.

9. Landis TR, Koch GG. The Measurement of Observer Agreement for Categorical Data. Biometrics 1977;33:159-174.

\section{Tables}

Table 1. List of immobilisation devices investigated

\begin{tabular}{ll}
\hline Device & Name and vendor \\
\hline Vacuum mattress 1 & RedVac VM0991X01, Kohlbrat \& Bunz, Radstadt, Austria \\
\hline Vacuum mattress 2 & Germa EasyFix, Ferno International, Wilmington, Ohio, USA \\
\hline Vacuum mattress 3 & Germa All in One Continental $90 \mathrm{~cm}$, Ferno International, Wilmington, Ohio, \\
& USA \\
\hline Trauma transfer & TraumaTransfer, Eson Comfort, Landeryd, Sweden \\
board & \\
\hline
\end{tabular}

Table 2. Assessment of artefacts category by two radiologists. 
Reviewer 1 /

Reviewer 1 /

Reviewer 2

Reviewer 2

No mattress or transfer board Reference

Reference

Transfer board

$1 / 1$

$1 / 1$

RedVac

Activated

$2 / 2$

$1 / 1$

RedVac

Activated

$2 / 2$

$1 / 2$

RedVac

Activated

$2 / 2$

$2 / 1$

RedVac

Deactivated

$2 / 2$

$1 / 1$

RedVac

Deactivated

$2 / 2$

$1 / 1$

RedVac

Deactivated

$2 / 2$

$1 / 1$

RedVac

Deactivated and

$2 / 2$

$1 / 1$

stretched

RedVac

Deactivated and

$2 / 2$

$1 / 1$

stretched

RedVac

Deactivated and

$2 / 2$

$1 / 1$

stretched

Germa All in One Continental

Activated

$3 / 2$

$2 / 2$

$90 \mathrm{~cm}$, Ferno

Germa All in One Continental

Activated

$2 / 2$

$2 / 3$

$90 \mathrm{~cm}$, Ferno

Germa All in One Continental

Activated

$3 / 3$

$3 / 2$

$90 \mathrm{~cm}$, Ferno

Germa All in One Continental

Deactivated

$1 / 1$

$1 / 1$

$90 \mathrm{~cm}$, Ferno

\begin{tabular}{llcr} 
Germa All in One Continental & Deactivated & $1 / 1$ & $2 / 2$ \\
$\mathbf{9 0} \mathbf{c m}$, Ferno & & & \\
\hline $\begin{array}{l}\text { Germa All in One Continental } \\
\mathbf{9 0} \mathbf{~ c m} \text { Ferno }\end{array}$ & Deactivated & $1 / 1$ & $1 / 1$ \\
\hline
\end{tabular}




\begin{tabular}{lccc} 
Germa All in One Continental & $\begin{array}{c}\text { Deactivated and } \\
\text { stretched }\end{array}$ & $2 / 1$ & $1 / 1$ \\
$\mathbf{9 0} \mathbf{~}$, Ferno & Deactivated and & $1 / 1$ & $1 / 1$ \\
Germa All in One Continental & stretched & & $1 / 1$ \\
$\mathbf{9 0} \mathbf{~ c m}$ Ferno & Deactivated and & $1 / 1$ & $3 / 3$ \\
\hline Germa All in One Continental & stretched & & $3 / 2$ \\
$\mathbf{9 0} \mathbf{c m}$, Ferno & Activated & $3 / 3$ & $3 / 3$ \\
\hline Germa EasyFix, Ferno & Activated & $3 / 3$ & $3 / 2$ \\
\hline Germa EasyFix, Ferno & Activated & $3 / 3$ & $2 / 2$ \\
\hline Germa EasyFix, Ferno & Deactivated & $2 / 2$ & $2 / 1$ \\
\hline Germa EasyFix, Ferno & Deactivated & $2 / 2$ & $2 / 1$ \\
\hline Germa EasyFix, Ferno & Deactivated & $2 / 2$ & $1 / 1$ \\
\hline Germa EasyFix, Ferno & Deactivated and & $2 / 2$ & $2 / 2$ \\
\hline Germa EasyFix, Ferno & stretched & & \\
\hline Germa EasyFix, Ferno & Deactivated and & $2 / 2$ & \\
\hline Germa EasyFix, Ferno & stretched & $2 / 2$ & \\
\hline & Deactivated and & stretched & \\
\hline
\end{tabular}

Legend table 2. The artefacts were categorized independently by the two radiologists as no artefacts (category 1), artefacts not hampering clinical judgment (category 2), artefacts hampering clinical judgment (category 3) and artefacts rendering the investigation unsuitable for clinical judgment (category 4).

\section{Figures}




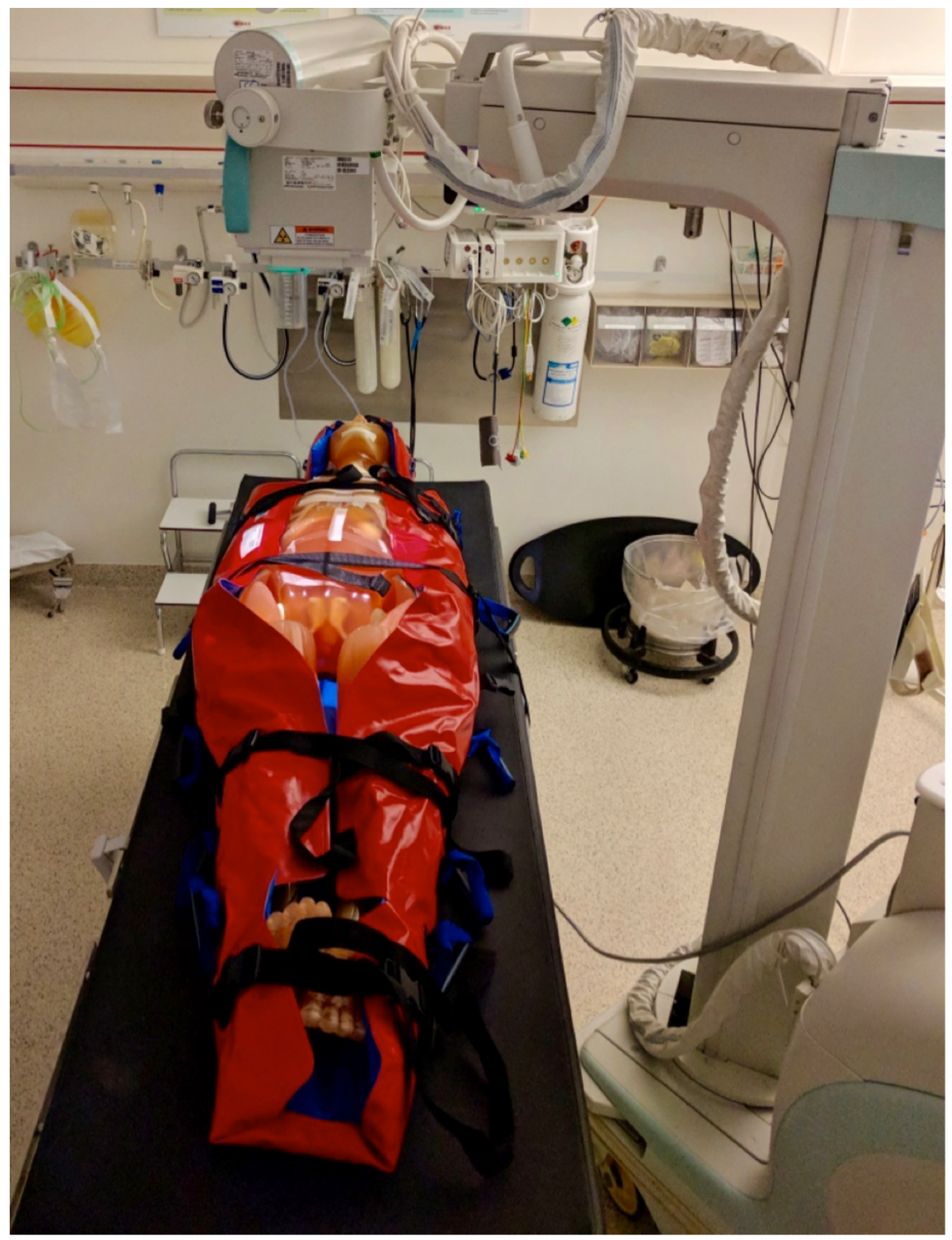

Figure 1

Phantom in activated vacuum mattress with straps ready for pelvic CR. 


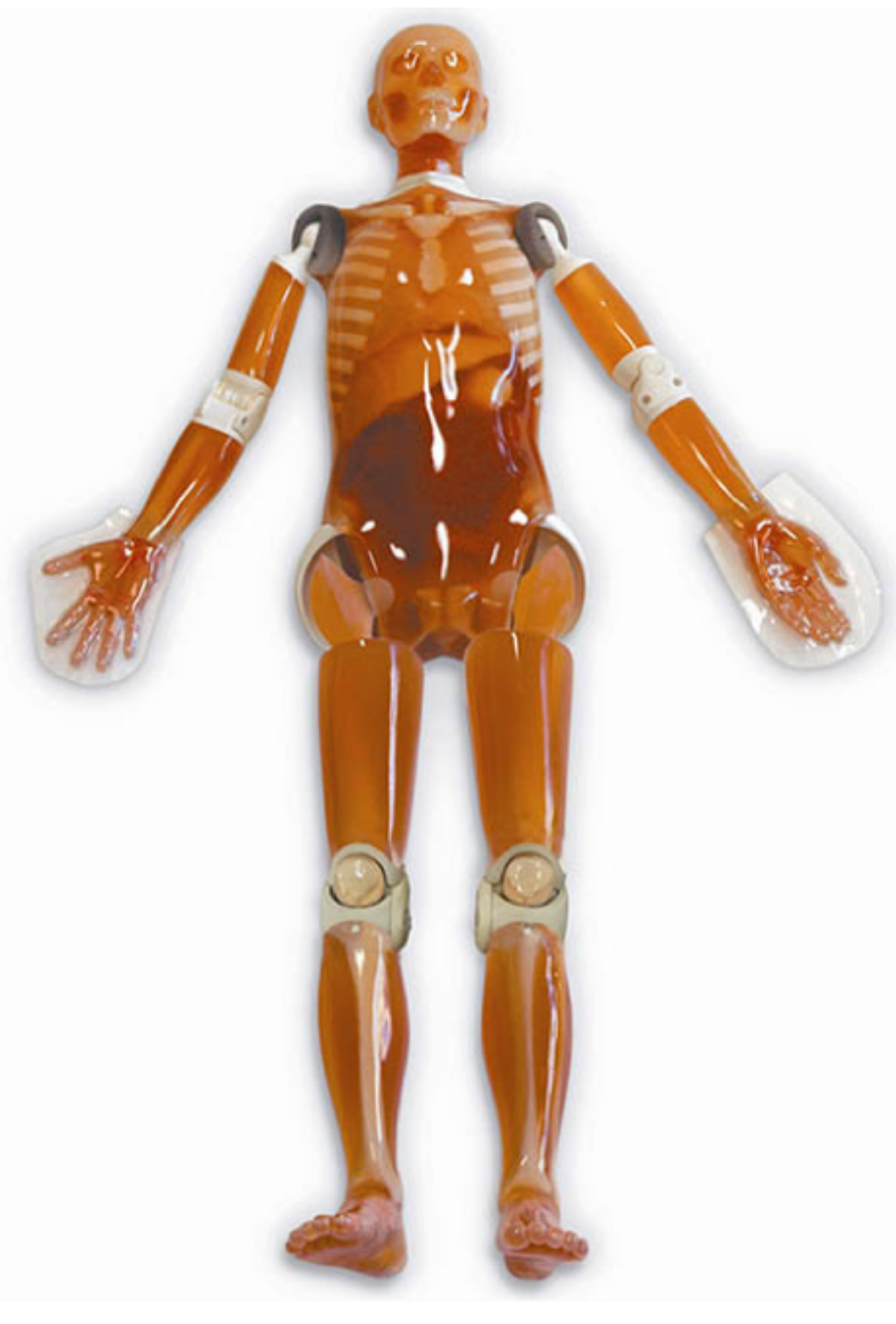

Figure 2

Anthropomorphic phantom PBU-60. (C) Kyoto Kagaku Co. LTD, Kyoto, Japan. 


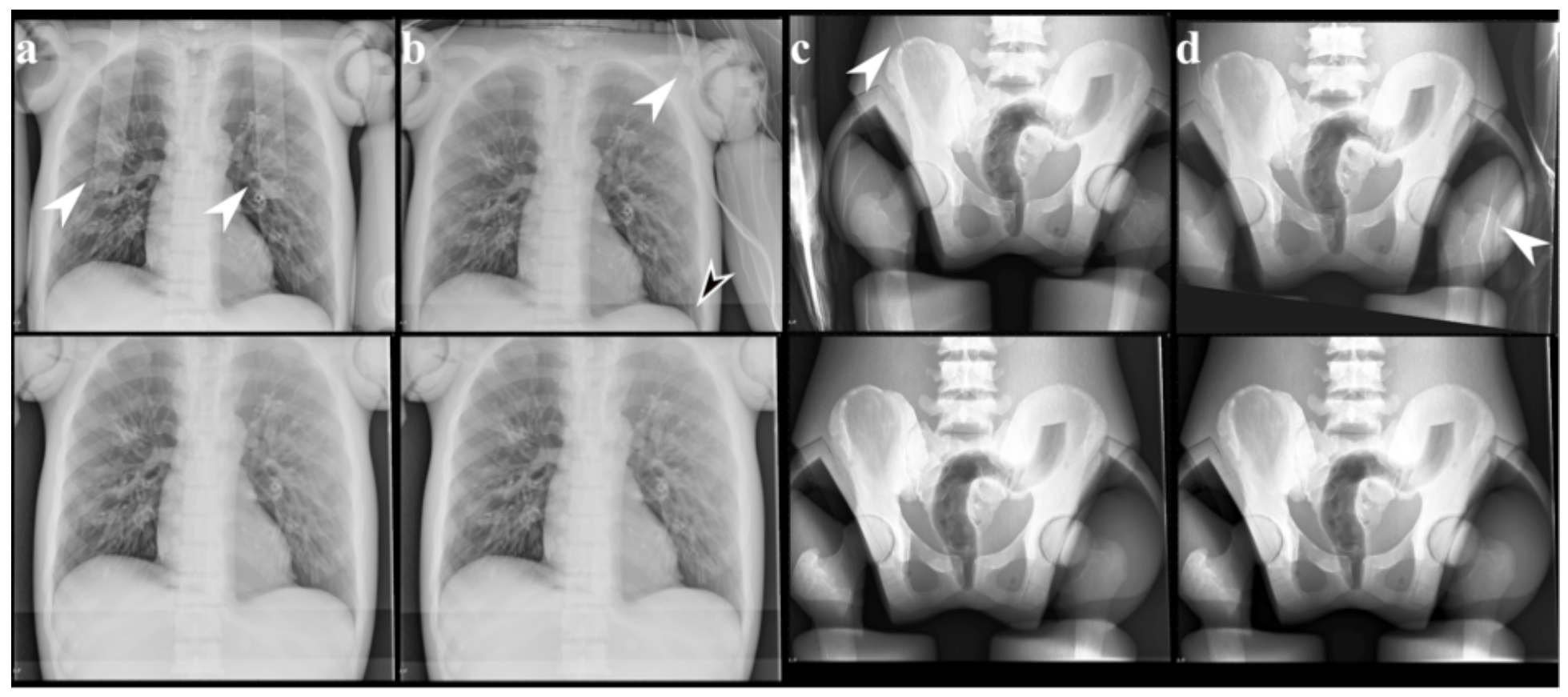

Figure 3

Examples of artefacts with reference images below. a. Chest category 2 artefact b. Chest category 2 (white arrow) and category 3 (black) artefacts. c. Pelvis category 2 artefact. d. Pelvis category 3 artefact.

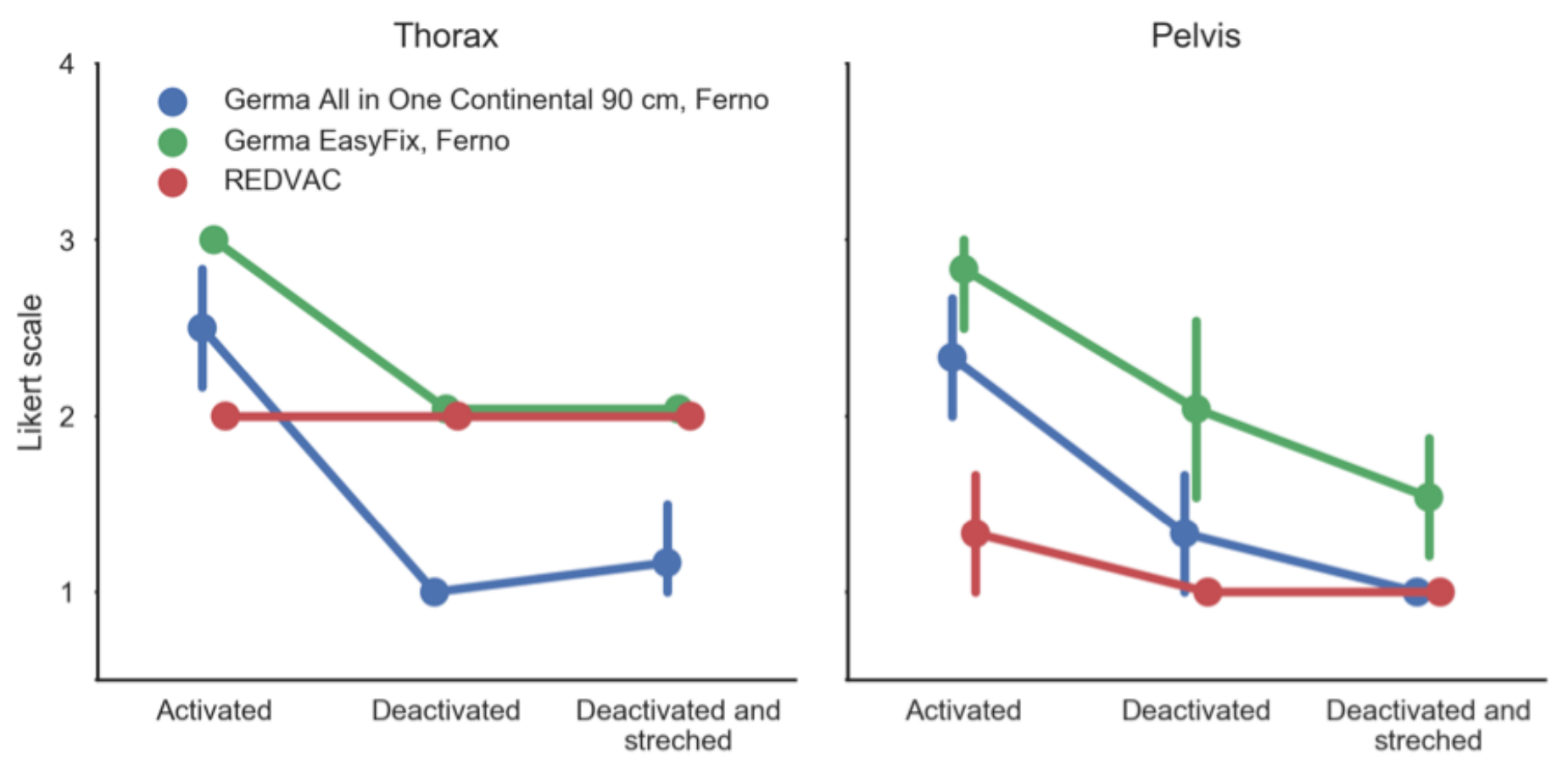

Figure 4

Assessment of image artefacts for three vacuum mattresses when activated, deactivated and stretched after deactivation. 